\title{
Riñón Poliquístico Infantil
}

\author{
Dra. Magaly Bamera V. ${ }^{\text {; }}$ Dra. Laura Quezada $\$ .2$ \\ Infantile Policystic Kidney
}

\begin{abstract}
Eight ases of Infantile Policystic kidney disease are presented. A new theory linking this pathology to congenital hepatic fibrosis as another type of presentation of the same entity is exposed and discussed. These cases gather all the clinical and laboratory features to be included under this denomination. The importance of renal ecography as a tool for an accurate diagnosis is stressed while the difficulties in the management of the frequent high level arterial hypertension is underlined. These findings are also described in the foreign-literature.

(Key words: Cystic Kidney. Policystic renal disease. Congenital hepatic fibrosis. Ecotomography. Arterial hypertension).
\end{abstract}

Las enfermedades que afectan al parénquima renal son muy variadas; entre ellas, los quistes ocupan un porcentaje no despreciable ${ }^{1}$. Estos pueden ser únicos o múltiples, gigantes o pequeños.

El Riñón Poliquistico de forma Infantil (RPI) es una enfermedad quistica renal que compromete ambos riñones, habitualmente en forma simétrica y que se trasmite por herencia autosómica recesiva. Afortunadamente es poco frecuente: $6,4 \times 100.000$ egresos hospitalarios 4 ; $4,24 \%$ de las masas abdominales de recién naci$\operatorname{dos}^{5}$ y $11,4 \%$ de las masas abdominales de origen renal en recién nacidos ${ }^{3}$, porque nos enfrenta a un problema grave desde el periodo neonatal, y tiene mal pronóstico $0^{2}, 3,4,5$.

A diferencia del anterior, el Rifón Poliquistjco de forma Adulta se trasmite por herencia autosómica dominante, los síntomas aparecen habitualmente en la tercera década de la vida (aunque pueden aparecer en recién nacidos) ${ }^{6,7}$ y su histología incluye dilatación de todo el nefrón, incluyendo la cápsula de Bowman.

\section{PACIENTES Y METODO}

Se revisaron los antecedentes de los pacientes del Hospital San Juan de Dios de Santiago, en quienes se ha confirmado el diagnóstico de Riñón

1. Médico del Departamento de Nefrología Infantil Hospital San Juan de Dios.

Infantil, Hospital San Juan de Dios.

2. Médico Becario de Pediatría, Hospital San Juan de Dios.
Poliquistico de forma Infantil. En la Tabla 1 se presenta nuestra experiencia que abarca los últimos 12 años, en los cuales hemos logrado reunir 8 casos, 3 de sexo masculino y 5 de sexo femenino; 6 de ellos tienen comprobación anatómica por necropsia y 2 se encuentran actual. mente en control, y su diagnóstico ha sido corroborado por estudios clínicos que incluyen pielografía, ecografia renal y cintigrafía renal y hepática compatibles con RPI.

\section{COMENTARIO}

La teoría etiopatogénica acerca del RPI ha cambiado desde aquella de Blyth y Ockenden en $1971^{B}$, quienes postularon que esta enfermedad sería un grupo heterogéneo de diferentes condiciones clínicas transmitidas con un mistno tipo de herencia. Esta teoría fue rebatida posterior. mente por evidencias histológicas que demostraron que la separación entre RPI neonatal, perinatal e infantil propiamente tal descritos por dichos autores, no existias. Lieberman, años más tarde 20,11 , aplica un criterio más unitario, pero aún establece una separación entre RPI y RPI asociado a Fibrosis Hepática Congénita. Este último es el compromiso hepático, habitualmente concomitante con el RPI. Según algunos autores citados por Lieberman, la frecuencia de este compromiso es variable entre 35 a $\mathbf{4 0 \% 1 0}$.

Finalmente, en 1976 , Bernstein y cols ${ }^{12}$ establecen que la diferenciación entre RPI y RPI más Fibrosis Hepática Congénita no existe. Ambas entidades serían dos componentes de una misma afección, sin una demarcación muy clara entre ellos ya que la FHC se asocia a otras malformaciones (renales o no) lo que la hace aparecer 
Tabla 1.

Riñón Poliquístico Infantil - Casuística Hospital San Juan de Dios (1972-1984).

\begin{tabular}{|c|c|c|c|c|c|c|}
\hline \multirow{2}{*}{\multicolumn{2}{|c|}{$\begin{array}{l}\text { CASO ANTECEDENTES } \\
\text { (SEXO) FAMILIARES }\end{array}$}} & \multirow[b]{2}{*}{ CLINICA } & \multicolumn{3}{|c|}{ DIAGNOSTICO } & \multirow[b]{2}{*}{ SEGUIMIENTO } \\
\hline & & & Pielo. & $\overline{\text { Eco. }}$ & Cint. & \\
\hline $\begin{array}{c}1 \\
\text { (M) }\end{array}$ & & $\begin{array}{l}\text { Sínd. Dific. respiratoria } \\
\text { Hipert, arterial } \\
\text { Retardo crecimiento }\end{array}$ & + & + & + & $\begin{array}{l}\text { En control } 13 \text { años } \\
\text { I.R.C. }\end{array}$ \\
\hline $\begin{array}{r}2 \\
\text { (F) }\end{array}$ & Desconocido & $\begin{array}{l}\text { Depresión-respiratoria } \\
\text { Síndr. dific. respiratoria } \\
\text { Hipert. arterial }\end{array}$ & + & + & + & $\begin{array}{l}\text { En control } 9 \text { meses } \\
\text { I.R.C. }\end{array}$ \\
\hline $\begin{array}{r}3 \\
(M)\end{array}$ & + & $\begin{array}{l}\text { Depresión neonatal severa } \\
\text { no recuperada }\end{array}$ & - & - & - & $\begin{array}{l}\text { Fallece } 2 \text { horas } \\
\text { RPI + FHC }\end{array}$ \\
\hline $\begin{array}{c}4 \\
(M)\end{array}$ & Desconocido & Sin antecedentes clínicos & - & - & - & $\begin{array}{l}\text { Diag. operatorio. } \\
\text { Fallece } 2 \text { días. }\end{array}$ \\
\hline $\begin{array}{r}5 \\
(\mathrm{~F})\end{array}$ & + & $\begin{array}{l}\text { Hipert. arterial } \\
\text { Reflujo gastroesofágico }\end{array}$ & + & - & + & $\begin{array}{l}\text { Fallece } 3 \text { meses. } \\
\text { RPI + FHC }\end{array}$ \\
\hline $\begin{array}{r}6 \\
(F)\end{array}$ & Dudoso * & $\begin{array}{l}\text { Sind. dific. Iespiratoria } \\
\text { Neumomediastino } \\
\text { Hipert. arterial } \\
\text { Reflujo gastroesofágico }\end{array}$ & + & + & + & $\begin{array}{l}\text { Fallece } 14 \text { meses. } \\
\text { RPI + FHC }\end{array}$ \\
\hline $\begin{array}{r}7 \\
(\mathrm{~F})\end{array}$ & + & $\begin{array}{l}\text { Sind. dific. respiratoria } \\
\text { Hipert. arterial } \\
\text { Infección urinaria }\end{array}$ & + & + & - & $\begin{array}{l}\text { Fallece } 25 \text { dias. } \\
\text { RPI + FHC }\end{array}$ \\
\hline $\begin{array}{r}8 \\
(\mathrm{~F})\end{array}$ & Desconocido & $\begin{array}{l}\text { Vómitos intensos. } \\
\text { Hiponatremia }\end{array}$ & - & - & & $\begin{array}{l}\text { Fallece } 2 \text { meses. } \\
\text { RPI + FHC }\end{array}$ \\
\hline
\end{tabular}

(M) = Sexo Masculino

(F) = Sexo Femenino

IRC = Insuficiencia Renal Crónica
RPI = Riñón Poliquístico Infantil

FHC = Fibrosis Hepática Congénita

* $\quad$ = Padre con hematuria períodica, pero rechaza estudio clínico como una manifestación más de un problema y no como una entidad aparente.

Además no hay diferencias histológicas ni en la microdisección entre los casos catalogados como RPJ "puros" y los asociados a FHC. La histologia hepática y renal de ambos grupos se caracteriza por gran dilatación tubular medular y en mayor proporción de los túbulos colectores; la unión del túbulo colector con el túbulo contorneado distal es normal; no hay elementos displásicos en el parénquima renal; el daño renal es progresivo y se caracteriza por fibrosis peritubular; el daño hepático se caracteriza por fibrosis periportal y dilatación de los conductillos biliares.

En base a esto se plantea que la explicación para todo este dafo podría estar en una falla genética que determine una alteración primaria de las estructuras de sostén de los epitelios o directamente a las proteinas de los epitelios ${ }^{12}$. Esto permite ligar la alteración renal con el compromiso de otros parénquimas a través de fallas enzimáticas comunes ${ }^{13}$.

Clínicamente ${ }^{\mathbf{1 0}}$, estos casos pueden ser divididos en dos grupos: El de los RPI con riñones gigantes en el período de recién nacido, y el de los RPI def niño mayor que presenta gran heterogeneidad clínica.

Esta diferenciación sirve sólo al clínico y es una manifestación de la evolución espontánea de la enfermedad, que es variable a lo largo del tiempo.

Así, tenemos que la clínica del recién nacido está comandada por el trastorno respiratorio con neumornediastino, neumotórax o ambos; atelectasia pulmonar; hipertensión arterial severa; falla renal y grandes masas abdominales palpables.

En el periodo de lactante, la clínica toma un matiz diferente ya que la hipertensión arterial adqujere relevancia y también los procesos infecciosos pulmonares. 
En el preescolar y escolar la palpación renal se hace más difícil, la hipertensión arterial más manejable, se acentúa la insuficiencia renal con el consiguiente retraso del crecimiento y aparece, en los niflos mayores, la hipertensión portal que se expresa en várices esofágicas y esplenomegalia, evidenciándose asi el creciente compromiso hepático de la FHC.

Nuestros casos cumplen casi todos los requisitos clínicos exigjdos en la literatura. Hay que recalcar que la hipertensión arterial es uno de los elementos de más difícil manejo en esta enfermedad, hecho que también se encuentra descrito por otros autores, sin que se conozca el mecanismo patogénico involucrado 10.11 .12$.

Se piensa que esta enfermedad se transmite por herencia autosómica recesiva. En nuestra casuistica hay antecedentes comprobados en 4 casos; el paciente númeto 7 es el tercer hermano fallecido por esta causa (uno de ellos no está incluido por no tener estudio clínico ni necrópsico, pero la palpación de grandes masas abdominales en el período de recién nacido hacían muy probable el diagnóstico: falleció a las 48 horas de vida).

Todos nuestros pacientes fueron diagnosticados por haberse palpado masas abdominales al nacer. Actualmente sobreviven 2 niños, de 13 años y 9 meses respectivamente, cursando este último con hipertensión arterial de muy dificil manejo.

Dentro del estudio se incluye pielografía de eliminación, la que muestra imágenes de ectasia tubular medular, con dilataciones que pueden ser saculares o cilíndricas, pero manteriendo el molde de la arquitectura renal; estas dilataciones se pierden un poco en los niños mayores, ya sea por disminución en el tamaño de los quistes o por aumento de la fibrosis peritubular 10,12 .

Los diagnósticos fueron hechos mediante pielografía, cintigrafía renal y hepática y, en los últimos casos, por ecografía renal. En los 6 pacientes en que se realizó necropsia, la histología renal era típica y todos presentaron además e] compromiso hepático caracteristico.

Actualmente el estudio ecográfico obstétrico permite el diagnóstico prenatal de masa abdomi. nal y la preparación para un parto eventualmente difícil por el tamaño renal ${ }^{24}, 15,16$. La ecogra. fía renal es también el método de mayor rendimiento para el estudio posterior del paciente $y$ de sus familiares en tiesgo $17,18,19,20,21$.

Suponemos que los enfermos que sobreviven se verán enfrentados a un deterioro progresivo de la función renal. Aún cuando la insuficiencia renal crónica se desarrolla desde muy temprano. su curso es variable, permitiendo en algunos casos alcanzar edades susceptibles de transplante renal, que es la solución al problema renal, aún cuando posteriormente presenten las complicaciones de Ja FHC con hipertensión portal. Este hecho hace aconsejable la realización de derivación porto-cava antes del transplante renal o muy cerca de éste 22,23 .

Los éxitos logrados en otros Centros con este manejo, en los niños que han logrado superar los dificiles primeros años, nos hacen abrigar espe. ranzas de solución para este poco frecuente pero grave problema.

\section{RESUMEN}

Se presentan 8 casos de Rifón Poliquístico Infantil y se expone la nueva teoría que lo liga a la fibrosis hepática congénita, situando ambas entidades como dos elementos de un mismo cuadro clínico. Los casos presentados reúnen todas las características clínicas y de laboratorio requeridas para el diagnóstico recomendándose la ecografia renal para efectuarlo.

Se destaca la magnitud de la hipertensión arterial, de muy difícil m̦anejo, hecho también consignado en la literatura.

\section{REFERENCIAS}

1. Mir S., Rapola J., Koskimies O.: "Reral cysts in pediatric autopsy material". Nephron 33: 189, 1983.

2. Kzzo $C_{\text {. }}$ Del Rio $A_{+,}$Laforre $J .:$ "Masas de flanco del recién nacido". Rev. Chîl. Pediat. 46: 139 , 1975.

3. Lzzo $C$.: "Tumores abdominales de origen renal en el recién nacido". En: Lagomarsino E. Nefrologia. Santiago. Andrés Bello, 1982. pp. 233-236.

4. Bruce $R_{\text {., Duckett } J . W .}$ "Cystic Disease of the Kidney, Dysplasia and Agenesis". En: Kelalis. Pediatric Urology. pp. 1159.

5. Wedge J., Grosfeld J., Smith J.: "Abdominal masses in the newborn: 63 cases". J. Urol. 105: 770, 1971.

6. Proesmans W., Van Damme B., Casaer P., Marchal $G$.: "Autosomal Dominant Polycystic Kidney disease in the neonatal period: Association with a cerebral arteriovenous malformation". Pediatrics 70: 971,1982 .

7. Anton P.A., Abramowsky C.R.: "Adult Polycystic Renal Disease presenting in infancy: a report emphasizing the bilateral involvement". J. Lrol. 128: $1290,1982$.

8. Resnick J., Vernier R.L.: "Cystic Disease of the Kidney in the Newborn Infant". Clin. Perinatol. 8 : 375,1981 .

9. Londing B.H., Wells T.R., Claireaux A.E.: "Morphometric analysis of the liver lesions in cystic disease of childhood". Hum. Pathol. 11 (Suppl); 549,1980 . 
10. Lieberman $E$.: "Management of the Child.with Cystic Disease of the Kidney". En: Lieberman E. Clinical Pediatric Nephrology. Philadelphia, Lippincott, 1976. pp. 340-371.

11. Liebenman E., Salinas-Madrigal L., Gwinn J.L., Brennan L.P., Fine R.N., Landing B.H.: "Infantile Polycystic Disease of the Kidneys and Liver: elinical, pathological and radiological correlations and comparison with Congenital Hepatic Fibrosis". Medicine $50277,1971$.

12. Bernstein J., Gardner X.D.: "Cystic Disease of the Kidney and Renal Dysplasia". En: Churg J. and Spargo B. Kidney Disease: Present Status. Williams \& Wilkins, New York, 1979, pp. 1399-1442.

13. Lehnert W., Wendel U., Lindenmaier S., Bohm $N$.: "Multiple acyl-Co A dehidrogenation deficiency (glutaric aciduria type II), congenital polycystic disease of the kidneys and symmetric warty dyspla. sia of the cerebral cortex in two brothers. Clinical, metabolical and biochemical findings". Eur $J$. Pediatr. 139: 56, 1982.

14. Legorth J., Verder H., Gronvall S.: "Prenatal Diagnosis of Multicystic Kidney by Uitrasound". Acta Obstet. Gyneool. Scand. 60: 523, 1981.

15. Netson L.H., Resnick M.I., Sumner T.E.: "Sonolucencies in fetal and infant abdomen: implications for management". Urology 15: 528, 1980.

16. Habif D.V. Jr, Berdon W.E., Yeh M.N.: "lnfantile Polycystic Kidney Disease: in utero sonographic diagnosis". Radiology 142: 475, 1982.

17. Rosenfield A.T., Lipson M.H., Wolf B., Taylor J.K. Rosenfield N.S., Hendler E.: "Ultrasonography and Nephrotomography in the presymptomatic diagnosis of dominantly inherited (adult-onset) polycystic kidney disease". Radiolony 135: 423, 1980.

18. Milutinovic J., Fialkow P.J., Phillips L.A., Agoda L.Y., Bryant J.I., Denney J.D., Rudd T.G.: "Autosomal Dominant Polycystic Kidney disease: early diagnosis and data for genetic counselling". Lancet $18180: 1203,1980$.

19 Berger P.E., Munschauer R.W., Kuhn J.P.: "Computed tornography and ultrasound of renal and perirenal diseases in infants and children. Relationship to excretory urography in renal cystic disease, trauma and neoplasm". Pediatr. Radiol. 9: $91,1980$.

20. Boal D.K., Teele R.L.: "Sonography of Infantile Polycystic Kidney Disease". A JR 135: 575, 1980.

21. Grossman $H .:$ "The evaluation of abdominal masses in children with emphasis on noninvasive methods". Cancer 35: 884, 1975.
22. Sanfilppo F.P., Vaughn W.K., Peters T.G., Bolinger R.R., Spees E.K.: "Transplantation for polycystic kidney disease". Transplantation 36: 139, 1983.

23. Mc Gonigle R.J., Mowat A.P., Bewick M., Howard E.R., Snowden S.A., Parsons V.: "Congenital hepatic fjbrosis and polycystic kidney disease: role of porta-caval shunting and transplantation in three patients". Q.J. Med. 50: 269, 1981.

24. Museles M., Gaudry C, Bason W.: "Renal anornalies in the newborn found by deep palpation" Pediatrics 47: 97,1971

25. Amner E.D., Ellis D., Jaffe R., Bowen A.: "Neonatal radiocontrast nephropathy simulating infantile polycystic kidney disease". J. Pedjatr. 100: 85, 1982.

26. Kuhn J.P., Berger P.E.: "Computed tomography of the kidney in infancy and childhood" Radiol. Clin. North. Am. 19: 445. 1981.

27. Mittelstaedt C,A, Volberg F,M., Fischer G.J., Mc Cariney W.H.: "Caroli's diseases: sonographic findings". AJR 134: 585, 1980.

28. Anselet P., Sabria M., Bartolomé J., Campos J, Olmos A., Piera L.: "Estudio clínico y evolutivo de 72 casos de poliquistosis renal del adulto". Rev. Clin. Esp. 160: 315, 1981.

29. Siapleton F.B., Bilton S., Wilcox J., Leopold G.R.: "Transient nephromegaly simulating infantile polycystic disease of the kidneys". Pediatrics 67: 554, 1981.

30. Milutinovic J., Agadoa L. Y.: "Potential causes and pathogenesis in autosomal dominant polycystic kidney disease". Nephron 33: 139, 1983.

31. Alvarez F., Bernard O., Brunelle F., Hadchonel $M$. Leblanc A., Odievre M., Alagille D.: "Congenital hepatic fibrosis in children". J. Pediatr. 99: 370, 1981.

32. Milutinov J., Fialkow P.J., Rudd T.G., Agodoa L.Y., Phillips L.A., Bryant J.Y.: "Liver cysts in patients with autosomal dominant polycystic kidney disease". Am. J. Med. 68: 741, 1980.

33. Krensky A.M., Reddish"J.M., Teele R.L.. "Causes of increased renal echogenicity in pediatric patients". Pediatrics 72: 840, 1983.

34. Elder J.S., Klausmann P.G, Saders R. C., Jeffs R.D.: "Clinicopathological conference: Flank mass in a nconate". J. Lrol, 126: 94, 1981.

35. Milutinovic J., Agodoa L.C., Cutler R.E., Stricker G.E.: "Autosomal Dominant Polycystic Kidney Disease: early diagnosis and consideration of pathogenèsis". Am. J. Clin. Pathol. 73: 740, 1980. 